\title{
Research on Application of Hospital Earthquake Early Warning System
}

\author{
ChangXian Zhou, ShaoPeng Zheng, YouQuan Ye, PuChun Li \\ Xiamen Seismic Survey Research Center, Xiamen 361021,China \\ 41500619-1@qq.com
}

Keywords: Earthquake Early Warning System, Hospitals, Early Warning Applications

\begin{abstract}
Earthquake early warning system is a new means to effectively reduce the earthquake disaster. There are many countries and territories in the world have developed a number of specific facilities, a single city or even larger areas of the earthquake early warning system, and some earthquake warning system has been formal for the public to release the earthquake warning information, and some are also on-line test operation. With the expansion of the seismic network, EEW method to improve the people's awareness of earthquake disaster reduction and community disaster reduction needs, earthquake early warning system has been rapid development. The earthquake early warning system is the complex social system engineering, not only has many complicated scientific and technical problems to be solved, but also need innovative social management thinking. This paper introduces the application of hospital earthquake early warning system, earthquake early warning system research and related application.
\end{abstract}

\section{Theoretical Introduction of Earthquake Warning System}

Earthquake warning system is based on the electromagnetic wave propagation speed is much greater than the speed of ground motion and the destruction of destructive ground motion is less than the early arrival of the less destructive $\mathrm{P}$ wave velocity of the basic principles of the use of high seismic activity in the region set up real-time The observation data of the seismic observation station is transmitted, and the information of the location of the earthquake, the magnitude of the magnitude and the possible earthquake field are quickly determined within a short time after the earthquake, and the earthquake warning information is released to the people in the target area in time.

The Earthquake Warning System is a system that can quickly detect earthquakes and warn of earthquakes in the event of an earthquake. In general, it uses a sound or image or both warnings to indicate a strongly shaking of seismic waves. Within a few seconds is coming. There are two kinds of early warning methods for earthquake early warning system, one is the early warning of seismic parameters and the other is the earthquake dynamic value warning. Seismic parameter warning The use of station $\mathrm{P}$ wave or $\mathrm{S}$ wave to determine the magnitude, source depth, epicenter and other parameters, to determine the scope and level of warning. In addition, according to the distance from the early warning target area can be divided into pre-earthquake warning and local $\mathrm{P}$ wave pre-earthquake ${ }^{[1]}$.

Earthquake warning technology applied to the actual formation of the earthquake early warning system. The basic hardware components of the earthquake early warning system are: earthquake monitoring system (digital real-time strong earthquake), communication system (telephone line, wireless dial-up, station), control center (computer and professional software) bucket alarm release system (mobile phone, electronic media, government). The full set of seismic warning systems should be characterized by a high degree of integration, real-time monitoring, rapid response, especially rapid response. Because the earthquake early warning system is in fact with the earthquake wave race, more outperforming $1 \mathrm{~s}$, will be able to get more than 1 s response time. 


\section{Important Role of Earthquake Early Warning System}

The length of the earthquake warning time depends on the distance from the early warning area to the epicenter distance. In addition, the location of the early warning system deployment time also has a great impact. If there is a place where the earthquake occurred $100 \mathrm{~km}$ from a city, after the earthquake, the $\mathrm{P}$ wave arrives after about 15 seconds, and the $\mathrm{S}$ wave reaches the wave after about $25 \mathrm{~s}$. If the city set up a set of equipment to detect seismic waves, theoretically there will be $10 \mathrm{~s}$ warning time. If the seismograph is set up further in the vicinity of the epicenter, the city will have a longer warning time ${ }^{[2]}$. Fig.1 shows schematic diagram of the principle of earthquake early warning.

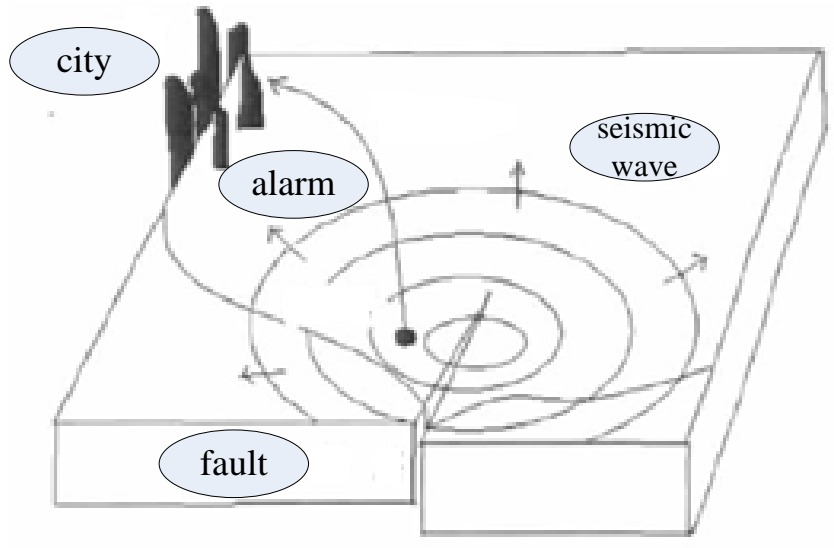

Fig. 1.Schematic diagram of the principle of earthquake early warning

At present, the earthquake prediction, forecast is still in the early stage of scientific exploration, and the overall level is still not high, especially the short-term and the level of earthquake prediction was still far apart. In contrast, the earthquake warning time is short, but its accuracy is relatively high, many countries have already begun to pay attention to the earthquake early warning system in the role of earthquake disaster reduction. For an area where effective warnings are available, there can be a lot of things to say in theory for a few seconds before the earthquake. For example: by automatic or manual way, the gas, electricity, water and other supplies to suspend, avoid the occurrence of secondary disasters such as fire; trains, cars and other means of transport can also be a short hedge time; primary and secondary schools or residential cottage. Where people can go outdoors; high-rise buildings can also find safe corner as soon as possible to avoid safe.

At present, the earthquake early warning system is still in the cradle stage, but the development of such a system is still very effective. Theoretically predicts the number of casualties that can be reduced by the earthquake early warning system: if the warning time is $3 \mathrm{~s}$, the casualty ratio can be reduced by $14 \%$; if 10 s, the casualty ratio is reduced by $39 \%$; if it is $30 \mathrm{~s}$, the casualty ratio $\%$. The results of a real-time seismic information utilization protocol (REIC), a nonprofit organization in Tokyo, Japan, show that the number of deaths in the region will be reduced by $25 \%$ if the main earthquake area gets an alarm $2 \mathrm{~s}$ ahead of time; , The death toll will be reduced by $80 \%{ }^{[3]}$.

\section{Construction of Hospital Early Warning System}

The general work flow of the hospital earthquake warning system is: seismic monitoring points (mechanical seismograph, electronic seismograph, shock cabinet), communication system (telephone line, wireless dial-up Internet, relay station), control center (computer and special software), alarm release system, power supply system. According to the distance between the epicenter and the target area, the seismograph is installed in the potential source area, and the early warning target area is tens of kilometers away from the seismic source by the difference between the electromagnetic wave and the seismic wave. In the modern earthquake early warning system, this type of early warning system through the $\mathrm{S}$ wave to determine the various seismic parameters, the accuracy is better, but from the earthquake to the alarm release time is longer. Fig.2 shows the 
basic components of earthquake early warning system.

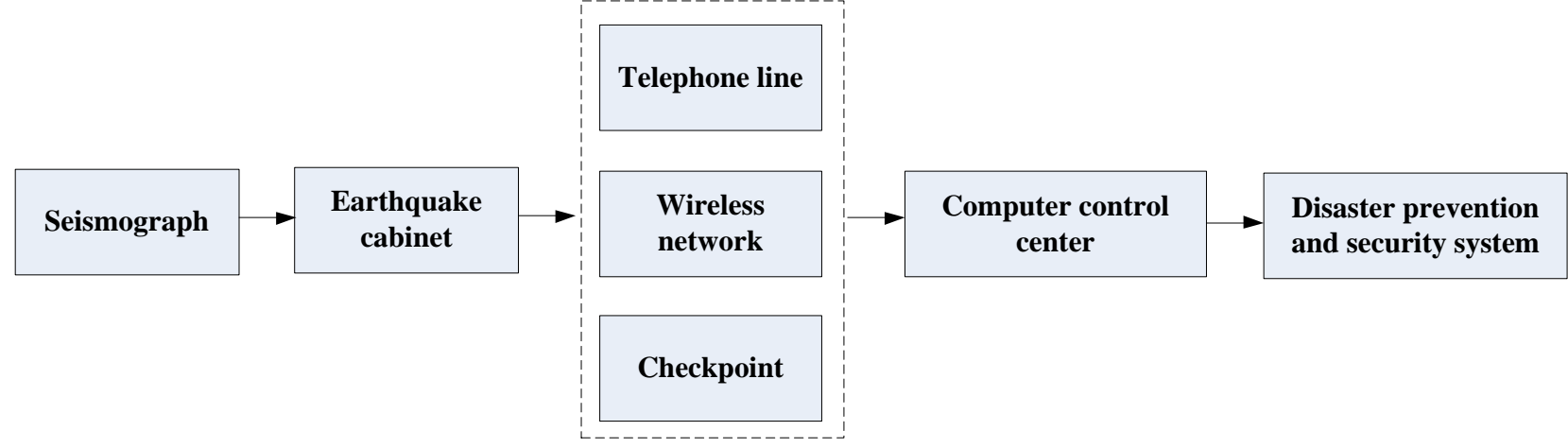

Fig.2.The basic components of earthquake early warning system

The structure of the hospital earthquake warning system should be designed according to the specific line conditions, but set the monitoring points in the station and along the traction substation. The seismic monitoring equipment consists of seismographs and seismographs, including seismographs and mechanical seismometers and electronic seismographs to improve safety. When the seismograph of the monitoring point monitors the acceleration of the earthquake equal to or greater than the alarm threshold, it will read the information measured by the neighboring monitoring points and verify the earthquake warning by verifying each other ${ }^{[4]}$.

The function of the earthquake early warning system also depends on the people's ability of earthquake disaster reduction and familiarity with the earthquake early warning system, which requires the relevant government departments to carry out various training and education activities to promote the people to the earthquake Early warning system, and can receive the most important emergency evacuation and hedging measures to receive self-help in the event of receiving the earthquake warning information, so that the functions of the earthquake early warning system can be the greatest play. Administrative law enforcement agencies should also be based on the actual situation to develop corresponding laws and regulations, clear the responsibility and obligations of earthquake warning information providers, and protect their legitimate rights.

\section{Application of Hospital Early Warning System}

The earthquake warning system not only succeeds in the rapid and accurate publication of the alarm information after the devastating earthquake, but also can raise the people's awareness of earthquake disaster prevention and mitigation, and finally achieve the purpose of effectively reducing the loss of earthquake disaster. The purpose of the medical system is to ensure that the EEW information is released as soon as possible, to ensure the safety of the patients, doctors, nurses and other staff, and to prevent accidental accidents during the operation, and to stop the operation of the equipment in medical tests. In addition, the research team developed a handbook for each type of employee in the demonstration hospital. Elevators, doors and emergency broadcasts should be controlled by an automatically enabled scheduled operation. For plant systems, EEW information must meet higher standards. Reducing the project's damage by stopping the production line can also reduce the recovery of production time. ${ }^{[5]}$.

On the other side, the wrong way to stop the production line will cause great damage to the factory. The hospital uses the earthquake warning and disaster prevention and disaster reduction multi-function equipment, and also includes a self-service rescue system, an earthquake warning device and a shock absorption system, the self-service rescue system is arranged on the headboard, the self-service and the rescue system are wirelessly connected with the hospital monitoring center, warning alarm set on the headboard, the earthquake warning alarm and the hospital monitoring center circuit. Hospital earthquake disaster reduction multi-function equipment, in addition to play the role of ordinary hospital bed rest, but also can play a role in the early warning of the earthquake, while the earthquake can be achieved through the earthquake to reduce the role of earthquake destructive power, and thus reduce the population-intensive hospital injuries. 
The success of foreign experience has led us to see through the scientific design and rational use of existing network resources to reduce the possibility of earthquake disasters, but also provides us with a new way of disaster reduction. With the continuous development of Chinese economic society, people demand for earthquake safety is increasing, so it is urgent to promote the construction of earthquake early warning system. The primary goal of the hospital is to ensure the safety of doctors and patients, followed by raising the level of education for disaster reduction. To this end, a special working group of experts was established to develop an emergency manual. In order to promote the application of emergency seismic information, through the training and exercise in the hospital, to reduce the earthquake caused by the loss and the annual education of about one million new students to make a great contribution.

\section{Conclusions}

Earthquake early warning system is an indispensable part of the disaster prevention monitoring system. At present, the rapid development of hospital construction in China, but the earthquake early warning system research has a considerable gap with the world's advanced countries. The early warning system of the earthquake can quickly detect the magnitude of the energy generated by the earthquake rupture and estimate that it will occur later in the local or off-site and in the event of strong earthquakes and warnings. The warning time ranges from a few seconds to a minute and its length depends on the distance between the early warning target and the epicenter of the earthquake. On the basis of introducing the basic principle of earthquake early warning technology, the present situation of earthquake early warning system is summarized and reviewed, which is of great significance for the research and construction of earthquake warning system in China.

\section{References}

[1] Nakamura Y. On-site alarm-the effective earthquake early warning [C].Fifth International Conference on Urban Earthquake Engineering, Tokyo, Japan, 2008.

[2] Mc Caffrey R. Global frequency of magnitude 9 earthquakes [J].Geology, 2008, 36; 263-266.

[3] Zhao Jidong. Development application and suggestions on earthquake early warning system [J]. geological evaluation of China, 2009, 28 (4):456-462.

[4] Allen R M, Uasparini P, Kamigaichi, Rose M. The status of earthquake early warning around the world introductory overview [J].Seismological research letters, 2009(b), 80(5): 682-693.

[5] Zollo A, Iannaccone U, Lancieri M, vt al. Earthquake early warning system in southern Italy methodologies and performance evaluation [J]. Geophysical research letters 2009, 36 ( LOOB07). 